\title{
Anticuerpos anti-Anaplasma spp en población de riesgo ocupacional de un hospital veterinario
}

\author{
ANTI- Anaplasma spp ANTIBODIES IN A POPULATION AT OCCUPATIONAL RISK OF A \\ VETERINARY HOSPITAL \\ Romy Weinborn A. ${ }^{*}$, Macarena Zanelli G. ${ }^{1}$, Óscar López S. ${ }^{1}$, Natalia Pau V. ${ }^{2}$, \\ Felipe Valdés P. ${ }^{1}$
}

\section{Resumen}

El estudio tuvo como objetivo estimar la seroprevalencia de anticuerpos contra Anaplasma spp en estudiantes y personal del Hospital Clínico Veterinario Docente (HCVD) de la Escuela de Medicina Veterinaria de la Universidad Santo Tomás (UST), Talca, Chile. Se analizaron 58 muestras sanguíneas humanas mediante inmunofluorescencia indirecta (IFI) para IgG. Se utilizó el test exacto de Fischer o $\mathrm{X}^{2}$ para evaluar diferencias significativas $(\mathrm{p}<0.05)$ y el índice de Kappa para evaluar concordancia entre IFI y signología clínica. Se obtuvo el $25.9 \%$ de seropositividad a Anaplasma spp, encontrándose diferencias significativas en uso de medidas de bioseguridad, extracción de garrapatas y hallazgos de ectoparásitos en ropa. El índice Kappa fue de 0.13.

Palabras clave: anaplasmosis; inmunofluorescencia indirecta; enfermedad ocupacional

\section{Abstract}

The objective of this study was to estimate the seroprevalence of antibodies against Anaplasma spp in students and staff of the Teaching Veterinary Clinical Hospital (HCVD) of the School of Veterinary Medicine of the University Santo Tomás (UST), Talca, Chile. Blood samples $(\mathrm{n}=58)$ were collected and analyzed by indirect immunofluorescence (IFI) for IgG. The exact Fischer or $\mathrm{X}^{2}$ test was used to evaluate significant differences $(\mathrm{p}<0.05)$ and the Kappa index to evaluate concordance between IFI and clinical signology. The

\footnotetext{
${ }^{1}$ Escuela de Medicina Veterinaria, Facultad de Recursos Naturales y Medicina Veterinaria, Universidad Santo Tomás, Talca, Chile

${ }^{2}$ Woodland Veterinary Hospital, Woodland, California, United States

${ }^{3}$ E-mail: rweinborn@santotomas.cl
} 
results showed $25.9 \%$ of seropositivity among students and staff for Anaplasma spp. Significant differences were found in the use of biosecurity measures, extraction of ticks, and ectoparasite findings in clothing. The Kappa index was 0.13 .

Key words: anaplasmosis; indirect immunofluorescence; occupational diseases

\section{INTRODUCCIÓN}

Las zoonosis son enfermedades que pueden ser naturalmente transmitidas de los animales al humano (Dabanch, 2003). Las ehrlichiosis son enfermedades zoonóticas y emergentes (Vu hai et al., 2014), causadas por bacterias Gram negativas e intracelulares obligadas. En humanos destacan Ehrlichia chaffensis y Anaplasma phagoytophilum (A. phagocytophilum) (Anaya et al., 2009; Gaowa et al., 2014). Debido a las reacciones cruzadas que existen entre rickettsias y ehrlichias (Oteo y Brouqui, 2005), A. phagocytophilum se referirá como Anaplasma spp en la presente investigación.

La infección en humanos y en perros se transmite por las garrapatas, las cuales succionan sangre a sus hospederos (Tamí y Tamí-Maury, 2004). El contacto con caninos infestados aumenta la posibilidad de adquirir esta enfermedad (Ural et al., 2014), manifestándose como un cuadro febril inespecífico (Anaya et al., 2009; Hernández-Ayazo y Marien-Clarete, 2013). Existen antecedentes a nivel mundial sobre estas zoonosis (Dolz et al., 2013; Breitcshwerdt et al., 2014; Kim et al., 2014; Silva et al., 2014; Yoshikawa et al., 2014) y antecedentes sobre poblaciones de riesgo como es el caso de los médicos veterinarios (Dowd, 2013; Irwin, 2014).

En Chile, al coexistir la presencia del vector (Rhipicephalus sanguineus) (López et al., 2003) con perros que deambulan por las calles con y sin propietarios, permitiría una mayor probabilidad de que el humano tenga contacto con el vector de la enfermedad, aumentando la posibilidad de zoonosis (Dabanch,
2003). En Chile existen pocos antecedentes de la enfermedad en humanos y caninos (Abarca et al., 2007, 2008; López et al., 2012), y solo ha sido descrita la presencia de anticuerpos contra Anaplasma spp en personas de la zona central de Chile con riesgo ocupacional; entre ellos, médicos veterinarios, peluqueros de canes y propietarios de canes (Abarca et al., 2008). En Talca no existen datos de anaplasmosis en humanos, aunque existen antecedentes para caninos, entre los que se pueden mencionar datos de ehrlichiosis en $16 \%$ en caninos con dueños atendidos en el Hospital Clínico Veterinario Docente de la Universidad Santo Tomás (Castillo, 2011), $48 \%$ de anaplasmosis en caninos vagabundos de la ciudad de Talca (Ortiz, 2012) y de $6 \%$ en perros atendidos en clínicas de la ciudad de Talca (Benavides, 2015), motivo por el cual existirían poblaciones con riesgo ocupacional. Es por ello que se desarrolló esta investigación con el objetivo de estimar la seroprevalencia de anticuerpos contra Anaplasma spp en personal del Hospital Clínico Veterinario Docente (HCVD) de la Universidad Santo Tomás (UST) en Talca, Chile.

\section{Materiales y Métodos}

\section{Tamaño Muestral y Procedimiento de Muestreo}

El estudio se llevó a cabo en el Hospital Clínico Veterinario Docente (HCDV) de la Universidad Santo Tomás (UST), Talca, ubicado a 250 kilómetros al sur de la capital Santiago de Chile. Como criterio de inclusión se consideraron alumnos y trabajadores que participaban en actividades diarias del HCVD. 
Los participantes fueron elegidos aleatoriamente y contestaron una encuesta epidemiológica.

Se recolectó sangre entera de la vena cefálica en tubos para la obtención de suero en agosto de 2013 en el Laboratorio de Técnico en Enfermería y en el HCVD, ambos de la UST. Las muestras fueron centrifugadas a $12096 \mathrm{~g}$ por 5 minutos. El sobrenadante fue congelado a $-70{ }^{\circ} \mathrm{C}$ hasta su procesamiento, el cual se realizó en el Laboratorio de Patología Animal de la UST Santiago, por medio de inmunofluorescencia indirecta para detección de anticuerpos IgG anti A. phagocytophilum (Fuller Laboratories, EEG-120, EEUU), siguiendo las indicaciones del fabricante, considerando como positivos aquellos positivos a la dilución 1/64.

El tamaño muestral $(n=58)$ se calculó mediante la fórmula de poblaciones finitas, con una población inicial de 63 personas, con seguridad del 95\% $\left(Z_{a}=1.96^{2}\right)$, proporción esperada del $18.5 \%(\mathrm{n}=11)$, además, $\mathrm{q}=1-\mathrm{p}$ $(0.95)$ y precisión $(\mathrm{d}=3 \%)$.

\section{Consideraciones Éticas}

Las muestras fueron recolectadas por personal capacitado, considerando las normas requeridas para la investigación en humanos, Ley 20584/2012 que regula los derechos y deberes que tienen las personas en relación con acciones vinculadas a su atención en salud, con confidencialidad de personas muestreadas y resultados (Diario Oficial de la República de Chile, 2012). El estudio fue autorizado por el Comité de Ética de la UST (Resolución N. ${ }^{\circ} 25 / 2013$ ). Así mismo, se solicitó a cada participante la firma de un consentimiento informado previo a la toma de muestra.

\section{Análisis Estadístico}

El estudio fue de tipo descriptivo y transversal. Los resultados fueron expresados en frecuencias relativas y absolutas. Se utilizó el test exacto de Fischer o $\mathrm{X}^{2}$, consi- derando un valor $\mathrm{p}<0.05$ como estadísticamente significativo.

Para el análisis de sintomatología fue considerado como sugerente la presentación de alguno de los siguientes síntomas: cefalea, fiebre, artralgia, mialgia o linfoadenopatía periférica. Para la concordancia se utilizó el índice Kappa, considerándose los resultados como insignificante (0-0.2), débil (0.21-0.4), moderado (0.41-0.60), bueno $(0.61-0.80)$ y muy bueno (0.81-1.00).

\section{Resultados}

Los resultados indicaron seropositividad del 25.9\% a Anaplasma spp (Cuadro 1). De los 15 seropositivos, 8 (53\%) eran mujeres y 7 (47\%) hombres. Del total de 43 seronegativos, $22(51 \%)$ fueron mujeres y $21(49 \%)$ hombres, no presentándose diferencias significativas entre géneros.

En el Cuadro 2 se muestra la seropositividad de las personas para Anaplasma spp según el tipo de actividad desarrollada con su mascota. Destaca el caso de los dueños que bañaban y dormían con las mascotas (37.5\%). No existieron diferencias significativas entre seropositivos y seronegativos, con excepción de la actividad de dormir juntos y con aquellos que no tenían estos tipos de interacción con las mascotas ( $\mathrm{p}<0.05)$.

Todos los que usaban pinzas y guantes para la extracción de las garrapatas o aquellos que no participaban en la extracción de garrapatas fueron seronegativos para Anaplasma spp (Cuadro 3). Se encontró diferencia significativa entre seropositivos y seronegativos para el grupo que extraía las garrapatas con la mano sin guantes $(\mathrm{p}<0.05)$.

De los 15 sujetos seropositivos, 6(40\%) encontraron garrapatas en la ropa de trabajo, y de los 43 seronegativos, 6 (14\%) lo reportaron (Cuadro 4). Se encontró diferencia significativa entre personas con y sin garrapatas en la ropa de trabajo en el grupo seronegativo $(\mathrm{p}<0.05)$. 
Cuadro 1. Frecuencia de casos positivos de Anaplasma spp en el Hospital Clínico Veterinario Docente de la Escuela de Medicina Veterinaria, Universidad Santo Tomás, sede Talca

\begin{tabular}{lccc}
\hline & $\begin{array}{c}\text { Población } \\
(\mathrm{n})\end{array}$ & $\begin{array}{c}\text { Seropositivo } \\
(\%)\end{array}$ & $\begin{array}{c}\text { Seronegativo } \\
(\%)\end{array}$ \\
\hline Estudiantes & 43 & 20.9 & 79.1 \\
Personal médico & 14 & 42.8 & 57.2 \\
Personal administrativo & 1 & 0 & 100 \\
\hline Total & 58 & 25.9 & 74.1 \\
\hline
\end{tabular}

Cuadro 2. Frecuencia de personal de la clínica veterinaria docente seropositivo y seronegativo a Anaplasma spp, según tipo de actividad desarrollada con su mascota

\begin{tabular}{lccc}
\hline & $\begin{array}{c}\text { Población } \\
(\mathrm{n})\end{array}$ & $\begin{array}{c}\text { Seropositivo } \\
(\%)\end{array}$ & $\begin{array}{c}\text { Seronegativo } \\
(\%)\end{array}$ \\
\hline Baño de mascotas & 13 & 15.4 & 88.6 \\
Dormir juntos & 4 & 25.0 & 75 \\
Bañar y dormir & 16 & 37.5 & 62.5 \\
Ninguna acción & 25 & 24.0 & 76 \\
\hline
\end{tabular}

Cuadro 3. Frecuencia de personal de la clínica veterinaria docente seropositivo a Anaplasma spp, según según el método utilizado para realizar extracción de garrapatas

\begin{tabular}{lccc}
\hline & $\begin{array}{c}\text { Población } \\
(\mathrm{n})\end{array}$ & $\begin{array}{c}\text { Seropositivo } \\
(\%)\end{array}$ & $\begin{array}{c}\text { Seronegativo } \\
(\%)\end{array}$ \\
\hline Mano sin guantes & 22 & 22.7 & 77.3 \\
Uso de pinzas & 28 & 35.7 & 64.3 \\
Pinzas y guantes & 1 & 0 & 100 \\
Ninguna acción & 7 & 0 & 100 \\
\hline
\end{tabular}

El $86 \%(50)$ de las personas encuestadas utilizaban medidas de bioseguridad al manipular al canino infeccioso, mientras que $8(14 \%)$ no lo hacían $(\mathrm{p}<0.05)$. Del grupo de seropositivos, $14(93.3 \%)$ aplicaba medidas de bioseguridad, mientras que $36(83.7 \%)$ de los seronegativos lo hacían ( $\mathrm{p}<0.05$; Cuadro 5). Además, de 15 sujetos seropositivos, 6
(40\%) declararon exposición a caninos con anaplasmosis, 5 (33\%) no habían tenido exposición y $4(27 \%)$ indicaron no saber. De los seronegativos, 4 (9\%) tuvieron esta exposición, 21 (49\%) no la tuvieron y 18 (42\%) no sabían, sin existir diferencias significativas entre estos grupos. 
Cuadro 4. Frecuencia de seropositividad a Anaplasma spp en personal de la clínica veterinaria docente, según el hallazgo de garrapatas en la ropa de trabajo

\begin{tabular}{lccc}
\hline & \multirow{2}{*}{$\begin{array}{c}\text { Personas } \\
\text { (n) }\end{array}$} & \multicolumn{2}{c}{$\begin{array}{c}\text { Garrapatas en } \\
\text { la ropa }\end{array}$} \\
\cline { 3 - 4 } & & Sí (n) & No (n) \\
\hline Seropositivos & 15 & 6 & 9 \\
Seronegativos & 43 & 6 & 37 \\
\hline
\end{tabular}

Cuadro 5. Frecuencia de seropositividad a Anaplasma spp en personal de la clínica veterinaria docente según utilización de medidas de bioseguridad

\begin{tabular}{lccc}
\hline & \multirow{2}{*}{$\begin{array}{c}\text { Personas } \\
\text { (n) }\end{array}$} & \multicolumn{2}{c}{$\begin{array}{c}\text { Medidas de } \\
\text { bioseguridad }\end{array}$} \\
\cline { 3 - 4 } & & Sí (n) & No (n) \\
\hline Seropositivos & 15 & 14 & 1 \\
Seronegativos & 43 & 36 & 7 \\
\hline
\end{tabular}

En cuanto a las manifestaciones clínicas compatibles a anaplasmosis, nueve sujetos seropositivos y 17 seronegativos tuvieron alguna signología clínica compatible con la enfermedad, sin evidenciar diferencias significativas. Entre los signos más frecuentes se encuentran la cefalea y la fiebre (Cuadro 6); sin embargo, al evaluar la concordancia entre los resultados de la prueba IFI y la signología sugerente a Anaplasma spp, el resultado del índice Kappa fue $(\mathrm{K})=0.13$, indicando que la concordancia fue considerada como insignificante.

\section{Discusión}

Los resultados indican seropositividad de $25.9 \%$ a $A$. phagocytophilum en estudian- tes y personal médico veterinario en el HCVD UST; datos concordantes con investigaciones realizadas en Chile, que indican que el principal grupo de riesgo para adquirir este tipo de patógeno es el personal médico, administrativo y de peluquerías de clínicas veterinarias (Abarca et al., 2008). Los resultados actuales son mayores al $18.5 \%$ descrito para Santiago de Chile (Abarca et al., 2008), posiblemente debido a que los propietarios de mascotas asistían a clínicas privadas en la capital, mientras que la población analizada en este estudio fueron personas que atendían mascotas de propietarios de estrato socioeconómico bajo, por ende, con menor posibilidad de realizar un efectivo tratamiento contra ectoparásitos (Weinborn, 2013). Además, la población estudiada en HCVD tiene mayor contacto diario con caninos con y sin dueño pudiendo, por lo tanto, tener un mayor contacto con ectoparásitos portadores del patógeno.

Reportes en Colombia señalan 20\% de seropositividad para anaplasmosis granulocítica humana (Máttar y Parra, 2006) y aunque ambos estudios comparten la técnica de laboratorio, existe la posibilidad que la prueba aplicada esté reconociendo otra bacteria, debido a las reacciones cruzadas que existen entre rickettsias y ehrlichias (Oteo y Brouqui, 2005).

El género de los sujetos seropositivos y seronegativos no presentó diferencias significativas, tal y como lo mencionan Tamí y Tamí-Maury (2004) en Venezuela; sin embargo, en España se reportan más casos en hombres que en mujeres (Oteo y Brouqui, 2005).

No fue posible evidenciar diferencias significativas con relación a actividades asociadas a las mascotas, a excepción en la actividad de dormir con las mascotas, coincidiendo con lo indicado en un estudio realizado en Polonia, en el que se establece que aumenta el riesgo de adquirir zoonosis al realizar esta actividad; sin embargo, esto tendría que asociarse a una inadecuada despa- 
Cuadro 6. Frecuencia de signos clínicos compatibles a Anaplasma spp en personal del Hospital Clínico Veterinario Docente de la Escuela de Medicina Veterinaria, Universidad Santo Tomás

\begin{tabular}{lcccccccccc}
\hline \multirow{2}{*}{$\begin{array}{l}\text { Sero- } \\
\text { positividad }\end{array}$} & \multicolumn{2}{c}{ Cefalea } & \multicolumn{2}{c}{ Fiebre } & \multicolumn{2}{c}{ Artralgia } & \multicolumn{2}{c}{ Mialgia } & \multicolumn{2}{c}{$\begin{array}{c}\text { Linfoadenopatía } \\
\text { periférica }\end{array}$} \\
\cline { 2 - 12 } & $\mathrm{n}$ & $\%$ & $\mathrm{n}$ & $\%$ & $\mathrm{n}$ & $\%$ & $\mathrm{n}$ & $\%$ & $\mathrm{n}$ & $\%$ \\
\hline Positivo & 8 & 33.3 & 7 & 63.7 & 4 & 50.0 & 7 & 58.3 & 4 & 66.6 \\
Negativo & 16 & 66.7 & 4 & 36.3 & 4 & 50.0 & 5 & 41.7 & 2 & 33.4 \\
\hline
\end{tabular}

rasitación (Kollataj et al., 2012). En el caso del HCVD, los estudiantes y el personal están versados sobre la tenencia responsable de mascotas, por lo que la propagación de zoonosis estaría limitada con medidas básicas de convivencia (Irwin, 2014).

Respecto a la extracción de ectoparásitos, solo un sujeto informó utilizar la técnica correcta de extracción; es decir, pinzas con mano enguantada (Oteo y Brouqui, 2005; Zhang et al., 2008); sin embargo, no se encontraron diferencias significativas en la mayoría de los casos, posiblemente debido a la baja frecuencia de garrapatas infectadas (Fernández, 2003). Es importante mencionar que ocurre ruptura tegumentaria durante la extracción, habiendo la posibilidad de contacto con sangre de animales infectados, lo que implicaría riesgo de zoonosis (Blanco et al., 2008).

Las personas encuestadas declararon no tomar las medidas de bioseguridad al trabajar con las mascotas, incluso teniendo caninos diagnosticados con la enfermedad. Por otra parte, es probable que exista un subdiagnóstico de esta enfermedad en caninos, ya que existen algunos antecedentes de ehrlichiosis y anaplasmosis, tanto en perros vagabundos como en caninos con dueños (Castillo, 2011; Ortiz, 2012; Benavides, 2015), por lo que siempre se recomienda como profilaxis utilizar equipo de protección personal adecuado e inspección visual corporal diaria en búsqueda de artrópodos (Oteo y Brouqui, 2005). La baja adherencia a medidas de bioseguridad por parte de la población estudiada podría aumentar el riesgo de enfermedad ocupacional (López et al., 2003; Máttar y Parra, 2006; Abarca et al., 2008). Por otra parte, se debe indicar que los trajes utilizados en la atención clínica no cubren completamente las extremidades (Smith et al., 2013).

La inespecificidad del cuadro clínico concuerda con los datos registrados en la literatura, donde se describe que la anaplasmosis se manifiesta con fiebre, malestar general y mialgias (Sainz et al., 2015). Asimismo, los datos obtenidos concuerdan con que es benigna en la mayoría de las personas, incluso asintomática o subclínica en los individuos más jóvenes (Fernández, 2003).

\section{Conclusiones}

La población participante del Hospital Clínico Veterinario Docente de la Universidad Santo Tomás, Chile presentó una prevalencia de $25.9 \%$ de anticuerpos contra Anaplasma spp. 


\section{Literatura Citada}

1. Abarca K, López J, González P, Dabanch J, Torres M, Solari V, Perret C. 2008. Evidencia seroepidemiológica de exposición humana a Anaplasma $s p$ en Santiago, Chile. Rev Chil Infectol 25: 358-361. doi: 10.4067/S071610182008000500008

2. Abarca K, Lopez J, Perret C, Guerrero J, Godoy P, Veloz A, et al. 2007. Anaplasma platys in dogs, Chile. Emer Infect Dis 13: 1392-1394. doi: 10.3201/ eid1309.070021

3. Anaya E, Morón C, Jaramillo K, Mendoza L, Román R. 2009. Evidencia serológica de ehrlichiosis humana en Ancash, Perú. Rev Peru Exp Salud Publica 26: 54-57. doi: 10.17843/ rpmesp.2009.261.1334

4. Benavides M. 2015. Detección de anticuerpos contra Anaplasma spp en 50 caninos atendidos en clínicas veterinarias de la ciudad de Talca, Región del Maule, Chile. Tesis de Pregrado. Talca: Univ. Santo Tomás. 38 p.

5. Blanco J, Jado I, Marín M, Sanfeliu I, Portillo A, Anda P, Pons I, et al. 2008. Diagnóstico microbiológico de las infecciones por patógenos bacterianos emergentes: Anaplasma, Bartonella, Rickettsia, Tropheryma whipplei. Enferm Infecc Microbiol Clin 26: 573580. doi: $10.1157 / 13128275$

6. Breitcshwerdt EB, Hegarty $B C$, Qurollo BA, Saito TB, Maggi RG, Blanton LS, Bouyer D. 2014. Intravascular persistence of Anaplasma platys, Ehrlichia chaffeensis, and Ehrlichia ewingii DNA in the blood of a dog and two family members. Parasit Vectors 7: 298. doi: 10.1186/1756-33057-298

7. Castillo D. 2011. Seroprevalencia de Ehrlichia canis mediante la técnica de ELISA (IMMUNOCOMB) en pacientes caninos del Hospital Clínico Veterinario Docente de la Universidad Santo Tomás de la ciudad de Talca, Región del
Maule, Chile. Tesis de Pregrado. Talca: Univ. Santo Tomás. 68 p.

8. Dabanch J. 2003. Zoonosis. Rev Chil Infectol 20 (Supl 1): S47-S51. doi: 10.4067/S0716-10182003020100008

9. Diario Oficial de la República de Chile. 2012. Ley de Derechos y Deberes del Paciente $\mathrm{N}^{\circ}$ 20.584. [Internet]. Disponible en: http://www.bucher.cl/ ley20584.pdf

10. Dolz G Ábrego L, Romero LE, Campos-Calderón L, Bouza-Mora L, Jiménez-Rocha AE. 2013. Ehrlichiosis y anaplasmosis en Costa Rica. Acta Méd Costarric 55(Supl 1): 34-40.

11. Dowd K, Taylor M, Toribio JA, Hooker C, Dhand NK. 2013. Zoonotic disease risk perception and infection control practices of Australian veterinarians: call for change in work culture. Prev Vet Med 111: 17-24. doi: 10.1016/j.prevetmed.2013.04.002

12. Fernández P. 2003. Garrapatas que parasitan a las personas en Castilla y León, determinación por serología de su parasitismo y detección moleculaza de los patógenos que albergan. Tesis Doctoral. Salamanca: Universidad de Salamanca. $295 \mathrm{p}$.

13. Gaowa YY, Ohashi $N, W u D$, Kawamori F, Ikegaya A, Watanabe T, Saitoh K, et al. 2014. Anaplasma phagocytophilum antibodies in humans, Japan, 2010-2011. Emerg Infect Dis 20: 508-509. doi: 10.3201/eid2003.131337

14. Hernández-Ayazo H, Marien-Clarete S. 2013. Entendiendo las ehrlichiosis humanas y destacando a un agente causal: Anaplasma phagocytophilum. Rev Cienc Biomed 4(1): 165-169.

15. Irwin PJ. 2014. It shouldn't happen to a $\operatorname{dog} \ldots$ or a veterinarian: clinical paradigms for canine vector-borne diseases. Trends Parasitol 30: 104-112. doi: 10.1016/j.pt.2013.12.001

16. Kim K, Yi J, Oh W, Kim N, Choi S, Choe P, et al. 2014. Human granulocytic anaplasmosis, South Korea, 2013. Emerg Infect Dis 20: 1708-1711. doi: 10.3201/eid2010.131680 
17. Kollataj W, Milczak A, Kollataj B, Karwat ID, Sygit M, Sygit K. 2012. Risk factors for the spread of parasitic zoonoses among dog owners and their families in rural areas. Ann Agric Envirom Med 19: 79-84.

18. López J, Abarca K, Mundaca MI, Caballero $C$, Valiente-Echeverría $F$. 2012. Identificación molecular de Ehrlichia canis en un canino de la ciudad de Arica, Chile. Rev Chil Infectol 29: 502-530. doi: 10.4067/S071610182012000600008

19. López J, Rivera M, Concha J, Gatica S, Loeffeholz M, Barriga O. 2003. Ehrlichiosis humana en Chile, evidencia serológica. Rev Méd Chile 131: 67-70. doi: 10.4067/S0034-98872003000100010

20. Máttar S, Parra M. 2006. Detection of antibodies to Anaplasma, Bartonella and Coxiella in rural inhabitants of the Caribean area of Colombia. Rev MVZ Córdoba 11: 781-789.

21. Oteo JA, Brouqui P. 2005. Ehrlichiosis $\mathrm{y}$ anaplasmosis humana. Enferm Infec Microbiol Clin 23: 375-380. doi: 10.1157/ 13076178

22. Ortiz M. 2012. Seroprevalencia de Ehrli-chia canis y Anaplasma phagocytophium en caninos vagabundos de dos sectores urbanos de la ciudad de Talca, Región del Maule, Chile. Tesis de Pregrado. Talca: Univ. Santo Tomás. 73 p.

23. Sainz A, Roura X, Miró G, EstradaPeña A, Kohn B, Harrus S, SolanoGallego L. 2015. Guideline for veteri-nary practitioners on canine ehrlichiosis and anaplamosis in Europe. Parasit Vectors 8: 75. doi: 10.1186/s13071-015-0649-0

24. Silva AB, Canseco SP, Gabriel de la Torre MP, Silva AM, Mayoral MA, Mayoral L, Pérez-Campos L, et al. 2014. Infección humana asintomática por contacto con perros. Un caso de ehrlichiosis humana. G Med Mex 150: 171-174.
25. Smith J, Packman Z, Hofmeister E. 2013. Multimodal evaluation of the effectiveness of a hand hygiene educational campaign at a small animal veterinary teaching hospital. J Am Vet Med Assoc 243: 1042-1048. doi: 10.2460/ javma.243.7.1042

26. Tamí IC, Tamí-Maury IM. 2004. Identificación morfológica de Ehrlichia sp en las plaquetas de pacientes con infección por virus de la inmunodeficiencia humana, en Venezuela. Rev Panam Salud Pública 16: 345-349. doi: 10.1590/S102049892004001100008

27. Ural K, Gultekin M, Atasoy A, Ulutas B. 2014. Spatial distribution of vector borne diseases agents in dogs in Aegean region, Turkey. Rev MVZ Córdoba 19: 4086-4098.

28. Vu hai V, Almeras L, Socolovschi C, Raoult D, Parola P, Pagès F. 2014. Monitoring human tick-borne disease risk and tick bite exposure in Europe: available tools and promising future methods. Ticks Tick Borne Dis 5: 607-619. doi: 10.1016/ j.ttbdis.2014.07.022

29. Weinborn R. 2013. Evaluación de las fichas clínicas como instrumento de apoyo a la práctica clínica y a la gestión del Hospital Clínico Veterinario Docente Universidad Santo Tomás Talca. Tesis de Magíster. Santiago: Univ. Santo Tomás. $100 \mathrm{p}$.

30. Yoshikawa Y, Ohashi O, Wu D, Kawamori F, Ikegaya A, Watanabe T, et al. 2014. Anaplasma phagocytophilum antibodies in humans, Japan 2010-2011. Emerg Infect Dis 20: 508509. doi: 10.3201/eid2003.131337

31. Zhang L, Liu Y, Ni D, Li Q, Yu Y, Yu $X$, Wan $K$, et al. 2008. Nosocomial transmission of human granulocytic anaplasmosis in China. JAMA 300: 2263-2270. doi: 10.1001/jama.2008.626 